\title{
Media-based Modulation: A New Approach to Wireless Transmission
}

\author{
Amir K. Khandani \\ E\&CE Department, University of Waterloo, Waterloo, ON, Canada
}

\begin{abstract}
It is shown that embedding part or all of the information in the (intentional) variations of the transmission media (end-to-end channel) can offer significant performance gains vs. traditional SISO, SIMO and MIMO systems, at the same time with a lower complexity. This is in contrast with the traditional wireless systems where the information is entirely embedded in the variations of an RF source prior to the antenna to propagate via the channel to the destination. In particular, it is shown that using a single transmit antenna and $D$ receive antennas; significant savings in energy with respect to a $D \times D$ traditional MIMO are achieved. Similar energy savings are possible in SISO, and SIMO setups.
\end{abstract}

\section{Introduction}

This work introduces the advantages of varying the end-toend channel, according to the input data (see Fig. 1), in a wireless communications system with multi-path fading. Such information baring channel variations are detected at the receiver end, resulting in an equivalent modulation scheme with Additive White Gaussian Noise (AWGN). In other words, the carrier is modulated after leaving the transmit antenna(s) by changing the Radio Frequency (RF) properties of the environment close to the transmitter, but external to the transmit antenna(s). This method of modulating the wave after leaving the transmit antenna(s), coined in this article as Media-Based Modulation (MBM), offers several advantages vs. traditional methods in which $\mathrm{RF}$ carrier is modulated prior to leaving the transmit antenna(s). In contrast with MBM, this article refers to the traditional methods as Source-Based Modulation (SBM).

MBM can be realized by changing RF properties, namely permittivity $(\varepsilon)$, and/or permeability $(\mu)$ and/or resistivity $(\rho)$, of the propagation environment close to the transmitter. It is well known that $\varepsilon, \mu, \rho$ appear in the Maxwell equations and consequently affect the corresponding solution. This in turn affects the end-to-end channel, and consequently the magnitude and phase of the received signal. Note that in a rich scattering environment, a small perturbation in the environment close to the transmitter will be augmented by many random reflections in the propagation path, resulting in an overall independent end-to-end channel realization.

If there are $2^{R_{m}}$ choices available for such channel perturbations, the corresponding received constellation will consist of $2^{R_{m}}$ points. It is obviously possible, and indeed desirable, to combine MBM and SBM by modulating the carrier partly prior to the transmission and partly afterwards. If an MBM of $2^{R_{m}}$ points is used together with an SBM of $2^{R_{s}}$ points, the overall constellation transmits $R_{s}+R_{m}$ bits which are channel coded to achieve reliable transmission over the underlying discrete input AWGN channel.

The idea of embedding information in varying a wireless channel is not new, and indeed precedes modern systems. In particular, Polybius came up with a system of alphabetical smoke signals around $150 \mathrm{BC}$. Mach-Zehnder modulators, widely used for signaling over fiber, modifies the light beam after leaving the laser. However, due to the lack of multipath in single mode fibers, the advantages discussed here in the context of wireless do not apply. The use of tunable parasitic elements/objects external to antenna for the purpose of beam forming is widely studied and practically realized in various forms. However, the objective in this class of works is "to focus/steer" the energy beam, which misses the advantages discussed here (when data is modulated by tuning such external parasitic elements/objects). There have been some recent works on embedding data in antenna beam-patters ${ }^{1}$. Alrabadi et al [1][2] discuss embedding phase information in orthogonal antenna patterns. However, this is motivated by reducing the number of transmit chains and no other benefits are discussed. Bains [3] discusses using parasitic elements for data modulation, and shows limited gains due to energy saving. However, the main features associated with such a setup (e.g., additive properties of information over multiple receive antennas) and methods to realize them, which are the sources of reported improvements in the current article, are not discussed. This article establishes the benefits of MBM, and methods to realize them. A similar analogy exists in the development of Multiple-Input Multiple-Output (MIMO) antenna systems, in the sense that the use of multiple antennas for beam forming was known, but the main advantages of MIMO in terms of spatial diversity and/or data multiplexing, and methods to realize them, were established in late 90's (see [4][5][6] and references therein).

Next, pros and cons of MBM vs. SBM are discussed.

A first advantage of MBM is in the increase of the number of received constellation points without increasing energy. To improve spectral efficiency, SBM alone should rely on using larger values for $R_{s}$, resulting in an exponential increase in transmit energy. In contrast, $R_{m}$ can be increased without directly affecting the transmit energy. Overall, $R_{S}$ and $R_{m}$ are selected to achieve the desired rate with the minimum transmit energy. Increase of $R_{s}$ can be, for example, achieved by modulating the carrier phase with $\pm \pi$ (changing the sign) and $\pm \pi / 2$ (exchanging the role of I and Q) to achieve a symmetrical constellation.

A second advantage of $\mathrm{MBM}$ is in its inherent diversity in dealing with slow fading. As the constellation points in MBM correspond to different channel realizations, unlike SBM, deep fades do not have a persisting effect. In other words, good and bad channel conditions contribute to a single constellation and span the entire constellation space. As a result, the spacing between constellation points is determined by relative values of different (low and high) complex channel gains (relative to each other). We refer to this feature as the Constellation Diversity. As the constellation size increases, this feature essentially converts a static multi-path fading channel into a non-fading AWGN

\footnotetext{
${ }^{1}$ Author is thankful to reviewers for pointing out refs [1][2][3].
} 
channel with effective signal energy equal to the received energy averaged over fading statistics. This feature, which occurs even in a SISO-MBM, is inherent and does not involve any tradeoffs. This is in contrast with the MIMOSBM where diversity over a static fading channel can be improved only at the cost of a reduction in rate [7].

A third advantage of MBM arises when the receiver has multiple antennas, namely in SIMO-MBM. In a $1 \times$ $D$ SIMO-SBM, the vector received over $D$ receive antennas spans a single complex dimension. Consequently, the effect of using multiple receive antennas is limited to energy saving which can be realized through maximum ratio combining. In contrast, for a $1 \times D$ SIMO-MBM, the received vector spans all the $\mathrm{D}$ receive dimensions. As a result, the spacing between received constellation points enjoys a scaling with the transmit energy similar to a SISOSBM with D times the bandwidth, or similar to a $D \times D$ MIMO-SBM. This feature mimics the multiplexing gain feature of MIMO [7].

A fourth advantage of MBM is in the independence of the noise components over multiple receiving antennas. Note that in both SBM and MBM, assuming transmitter uses uncorrelated code-books of equal energy over its transmit antennas, the statistics of the energy received per receive antenna does not depend on the number of receive antennas. This means, assuming $D$ receive antennas and a fading channel with a mean gain of one, $E$ units of transmit energy results in receiving $D E$ units of energy on the average. In the following, this feature is referred to as " $D$ times energy harvesting". Note that $1 \times D$ SIMO-MBM enjoys $D$ times energy harvesting, similar to that of $D \times D$ MIMO-SBM, and $1 \times D$ SIMO-SBM. The main promise of a $D \times D$ MIMOSBM is in providing an effect similar to that of $D$ parallel channels, with $D$ times energy harvesting. However, the performance of $D \times D$ MIMO-SBM falls short of such $D$ parallel channels as the MIMO channel matrix is typically non-orthogonal, or equivalently, noise components over information baring dimensions are dependent. This shortcoming is resolved in $1 \times D$ SIMO-MBM. In other words, one unit of transmit energy in a $1 \times D$ SIMO-MBM results in receiving, on the average, one unit of energy per receive antenna, while unlike $1 \times D$ SIMO-SBM, the energy received over each antenna constructs a new constellation. In other words, the received energy forms a constellation that spans the entire $D$ complex receive dimensions. This feature mimics the information scaling of a $D \times D$ MIMOSBM with multiplexing gain of $D$. This is in contrast with a $1 \times D$ SIMO-SBM in which the received energy spans a single complex dimension, allowing merely energy saving through receive beam forming (multiplexing gain is limited to one, regardless of $D$ ).

A fifth advantage of MBM is in the possibility of energy saving through selecting a subset of channel configurations, which results in a better overall performance for the given energy and spectral efficiency. This feature is similar to the so-called multi-user diversity gain in network scheduling.

A first disadvantage of MBM is that the arrangement of the constellation points is random and constellation points are used with equal probability, while in SBM, constellation points can be uniformly arranged, e.g. QAM (Quadrature
Amplitude Modulation) constellation, and can be used with non-uniform probabilities to realize some shaping gain. As it will be shown later, the degradation due to the random placement of the constellation points, and also due to using the points with equal probabilities, will be negligible as the constellation size increases.

A second disadvantage of MBM is that the transmitter is generally, although not necessarily, assumed to be oblivious to the modulation scheme, casting the problem into the class of transmission with outage. As will be shown later, the degradation due to the lack of transmit adaptation to channel will become negligible as the constellation size increases.

A third disadvantage of MBM is that the system is Linear, Time Varying (LTV), while SBM is Linear, Time Invariant (LTI). Unlike LTI, LTV systems can potentially expand the spectrum. Such a time varying feature also contradicts the functionality of the traditional channel equalization techniques. On the other hand, as the LTV nature of the system is due to the random selection (with equal probabilities) of one out of $M$ options for an underlying LTI channel, the power spectrum observed at any given receiver will be equal to the average of the power spectrums of the $M$ underlying LTI channels, times the input power spectrum. As wireless channel can transmit a wide range of frequencies, the underlying LTI systems will have a wide spectrum. As a result, the overall power spectrum follows the shape of the input spectrum (RF carrier prior to MBM). Another potential problem arises as traditional SBM systems exploit the LTI property and rely on some form of equalization to compensate the effect of the Inter Symbol Interference (ISI). Due to ISI, the energy associated with a single time symbol is spread over its neighbors. This means the signal over a single dimension at the input is spread over $L$ dimensions at the output, where $L$ denotes the length of the channel impulse response. In the case of SBM, the resulting $L$ dimensional output vector spans a single dimension, and equalization procedure should (ideally) accumulate the received energy corresponding to any given input signals (spread over these $L$ dimensions) into a separate decision variable. In contrast to SBM, in MBM, the $L$ dimensional vector at the channel output corresponding to a single transmission spans an $L$ dimensional space, increasing the information baring capability of any given time transmission by a factor of $L$. As a result, a transmission in MBM can be followed by $L$ zeros to flush out the channel memory and this is achieved without sacrificing the effective dimensionality of the overall signal space. An alterative would be to reduce the gap between successive transmissions to less than $L$ and apply sequence detection in time to account for the ISI. This can be achieved using a state diagram (evolving in time) with states corresponding to previous constellation points, or a quantized version of them to reduce the size of the state space. A more detailed analysis should include the effect of the channel impulse response for MBM, and the equalization for SBM.

\section{II- System Model and Relative Merits}

Figure 1 shows the setup of a $1 \times D$ SIMO-MBM. For the sake of simplicity, the concept is explained by focusing only on the MBM part of the transmission, and the combination 
with SBM is straightforward. There are $M=2^{R_{m}}$ messages, indexed by $m=1, \cdots, M$, which select one of the $M$ channel realizations corresponding to channel gains $\vec{h}(m)$ with components $h_{d}(m)$ for $d=1, \cdots, D$, and $m=1, \cdots, M$. Due to the normalization of fading, we have $E\left|h_{d}(m)\right|^{2}=1$ where $E$ denotes statistical averaging. AWGN vector $\vec{z}$ has independent identically distributed (i.i.d) components $z_{d}$, $d=1, \cdots D$, where $E\left|z_{d}\right|^{2}=\sigma^{2}$. In this setup, although the transmitter selects the channel realization $\vec{h}(m)$ to transmit message $m$, transmitter is oblivious to the details of $\vec{h}(m)$. On the other hand, the receiver knows $\vec{h}(m)$ and can perform signal detection, if the mutual information is sufficient. Receiver training is achieved by sending a set of pilots over different channel realizations, enabling the receiver to measure $h_{d}(m)$ for $\forall d, \forall m$. For a Raleigh fading channel (rich scattering), $h_{d}(m)$ are i.i.d Gaussian, which is in accordance with the optimality of Gaussian random coding over AWGN channels. However, as the transmitter is oblivious to the details of $\vec{h}(m)$, an outage may occur. A similar outage phenomenon exists in the case of SBM over a static fading channel which is handled by increasing the transmit energy, or by exploiting spatial diversity offered by using multiple antennas. However, in the case of SBM, this compensation necessitates a significant increase in the transmit energy, or loss in the rate by exploiting spatial diversity. Due to the inherent diversity of MBM, the issue of outage in slow fading channels will be much less problematic compared to SBM. As the noise is AWGN, we have:

$$
I(\vec{y} ; m)=I(\vec{y} ; \vec{h}(m))=H(\vec{y})-H(\vec{z})=H(\vec{y})-D \log _{2}\left(2 \pi e \sigma^{2}\right) .
$$

Although the maximum rate of such a transmission scheme is limited to $\log _{2} M$, as is the case in any channel with a discrete constellation of size $M$, the rate achievable in a proper operating point prior to the saturation can be significantly higher than its SBM counterpart. For example, next, MBM and SBM are compared in terms of the slope of rate vs. energy at low SNR. Recall that MBM and SBM are preferably combined, in which case due to symmetrical phase modulation in the underlying SBM portion, the resulting signal set will be symmetrical. Assuming a symmetrical signal set (obtained through sign change of the carrier to modulate an additional bit), for low values of Signal to Noise Ratio (SNR), we have [8]:

$$
\lim _{\alpha \rightarrow 0} \frac{I(\alpha)}{\alpha}=\frac{K G}{2} \text {, and } E\left(\lim _{\alpha \rightarrow 0} \frac{I(\alpha)}{\alpha}\right)=\frac{K^{2}}{2},
$$

where $I(\alpha)$ is the mutual information (for $\sigma^{2}$ normalized to one) as a function of a small increase in RF energy, namely $\alpha$, starting from $\alpha=0$ (zero RF energy), $G$ is the sample second moment of the $M$ constellation points, $K$ is the number of real dimensions, and $E($.$) denotes statistical$ expectation. Although $G$ is a random variable, its variance approaches zero with $1 / M$. This is in contrast to the case of a $D \times D$ MIMO-SBM for which the scaling of rate vs. SNR at low SNR at best (i.e., assuming feedback and water filling) is limited to the largest eigenvalue of the channel matrix. This means for low SNR values, a $D \times D$ MIMOSBM is essentially a one-dimensional channel with an energy gain corresponding to the largest eigenvalue. For a
Raleigh fading channel, these are eigenvalues of a $D \times$ $D$ random Wishart matrix for which the expected value of the largest eigenvalue, although being increasing with $D$, is limited to 4 which is approached as $D \rightarrow \infty$ [9]. In the case of a $D \times D$ MIMO-SBM, as SNR increases, water filling results in occupying more of the available dimensions. See Fig. 3.

In comparing MBM vs. SBM, to have a fair comparison, one should ideally compare the outage capacity of the two systems. However, a major benefit of MBM vs. SBM is that it changes the statistical behavior of the end-to-end channel, and consequently that of the mutual information, and in particular reduces its variance. This is due to the inherent diversity of MBM as each constellation point corresponds to a different channel realization and consequently good and bad channel conditions contribute to each transmission. This effect, which will be more pronounced at higher transmission rates, essentially converts a static fading channel into an Additive White Gaussian Noise (AWGN) channel where the SNR is determined by the received energy averaged over fading statistics. This is unlike MIMO-SBM where diversity over a static fading channel can be improved only at the cost of a reduction in spectral efficiency. In addition, such a comparison depends on the statistics of fading, and is further complicated by the interplay between rate and diversity order in MIMO-SBM. For these reasons, although being to the disadvantage of SIMO-MBM, the relative merits of MBM vs. SBM are studied in two different setups. A first setup, which focuses on the effect of the diversity inherent to MBM, primarily relies on SISO links and compares SISO-MBM vs. SISO-SBM in terms of outage capacity. A second setup, which focuses on the other features of MBM, primarily compares SIMO-MBM vs. MIMO-SBM in terms of Ergodic capacity.

Gain due to Inherent Constellation Diversity: Assuming Raleigh fading, the components of the constellation points are random and follow an i.i.d. Gaussian distribution. This is in agreement with random coding over an AWGN channel, however, the constellation structure in MBM remains the same over subsequent transmissions instead of having independent realizations as is required in random coding. This causes some loss in the achievable rate as compared to the capacity of the underlying AWGN channel, namely AWGN with a gain equal to the statistical average of fading. The achievable rate is a random variable (depends on the specific realization), fluctuating around the capacity of the underlying AWGN channel. As the number of constellation points increases, the variance of this random variable decreases, and the achievable rate will eventually tend to the capacity of the underlying AWGN channel. Let us normalize the statistical average of the Raleigh fading to one and power of the AWGN to $\sigma^{2}$, i.e. $\mathcal{N}\left(0, \sigma^{2}\right)$, and consider two ensembles of random codes. Ensemble I: An ensemble of cardinality $\mathcal{C}$ with i.i.d Gaussian components of variance one, i.e. $\mathcal{N}(0,1)$, over time and spatial dimensions. Ensemble II: A realization of the $M$ points constellation; i.e. a fixed set of $M$ points with i.i.d Gaussian components, its extension, and a random code selecting a subset of cardinality $\mathcal{C}$ in the extension. This is equivalent to using an i.i.d uniform distribution for random coding over the $M$-points constellation. Consider the 
collection of such random codes, each corresponding to a different realization of the $M$-points constellation. Let us select a codebook from each of these two ensembles, and for each code-word $\vec{x}$ in the codebook from Ensemble I, find the code-word $\overrightarrow{\hat{x}}$ in the codebook from Ensemble II that is at the minimum square distance to it. This is equivalent to quantizing $\vec{x}$ to $\overrightarrow{\hat{x}}$ using minimum mean square error. Let us denote the vector of quantization error by $\vec{c}$, i.e. $\vec{c}=\overrightarrow{\hat{x}}-\vec{x}$. Let us consider a communication system using the codebook from Ensemble I, achieving a rate of $0.5 \log _{2}\left(1+1 / \sigma^{2}\right)$ per real spatial dimension, i.e. a total rate of $0.5 D \log _{2}\left(1+1 / \sigma^{2}\right)$. The goal is to show that as $\mathrm{M} \rightarrow \infty$, this rate will be achievable by a $1 \times$ D SIMO-MBM. In other words, we have a random coding scheme using Gaussian code-words, but the code-word selected from this codebook should be quantized to its closet code-word from the codebook from ensemble II prior to transmission. Figure 1C shows the block diagram of such a system based on modeling the quantization error as additive noise $\vec{c}$. Components of $\vec{c}$ are i.i.d and correspond to the error terms in quantizing a sequence of $\mathcal{N}(0,1)$ Gaussian random numbers (code-word from the codebook of Ensemble II) with a set of $M$ points which are randomly generated using $\mathcal{N}(0,1)$. We have: $I(\vec{x} ; \vec{y})=H(\vec{y})-H(\vec{y} \mid \vec{x})$, and $H(\vec{y} \mid \vec{x}) \leq H(\overrightarrow{\hat{z}})$, consequently, $I(\vec{x} ; \vec{y}) \geq H(\vec{y})-H(\overrightarrow{\hat{z}})$. On the other hand, $H(\vec{y})-H(\overrightarrow{\hat{z}})$ is the capacity of additive independent noise channel in Fig. 1C. It is straightforward to show that as $M \rightarrow \infty$, mean and variance of $\|\vec{z}\|^{2}$ tend to zero. This means with probability one, capacity is equal to: $0.5 D \log _{2}\left(1+1 / \sigma^{2}\right)$ per real dimension. See [8] for more details.

\section{III- Numerical Comparisons}

Figure 4 primarily shows the effect of the constellation diversity and captures the relative performance of MBM vs. SBM in terms of outage behavior. Figure 5 shows the relative performance of MBM vs. SBM in terms of Ergodic capacity for SBM. This captures the effect of noise independence over receive dimensions for MBM. Figure 6 shows capacity of a 256 QAM in comparison with a random MBM constellation with 256 points. As seen in Fig. 6, the mutual information of a constellation with 256 points is relatively close to that of 256QAM constellation, with minor fluctuations (see the example shown in the sub-figure for a particular SNR of 15dB). Sub-figure in Fig. 6 shows that subject to about one dB of energy over-budgeting (as compared to AWGN), the SISOMBM will have a reliable performance, but the SISO-SBM requires $30 \mathrm{~dB}$ to $50 \mathrm{~dB}$ over-budgeting of SNR relative to the benchmark corresponding to AWGN channel. Figure 7 shows the effect of selecting a subset of a set of random points to maximize the mutual information subject to equal probability for the selected points (selection gain). As seen, a simple role based on selecting a subset of points with highest sample energy achieves a performance close to the best selection. See [8] for more details.

\section{IV-Candidates for RF Channel Perturbation}

Traditional RF beam forming schemes aim at concentrating energy in certain directions to increase received SNR. On the other hand, in MBM, the aim is to cause random variations in the received signal, without the need to know and/or control the imposed variations, neither to focus the energy. This objective is easier to realize as compared to traditional RF beam forming. In spite of these differences, many of the techniques developed for RF beam forming are applicable to MBM, including: Methods for changing property of a waveguide by surface plasma generated through light sources, or leaky wave antenna (based on a waveguide with tunable surface leakage). Creating surface plasma in an external to antenna parasitic object, e.g. using light intensity to change plasma depth causing a tunable impendence surface. Tuneable impedance surface as an external to antenna parasitic element, e.g. by changing the permeability of ferrite via a currentcarrying coil) changing the permittivity of ferroelectric material via a bias voltage, using meta-material as a parasitic object with changeable refraction index. More implementation details, and references, are provided in [8].

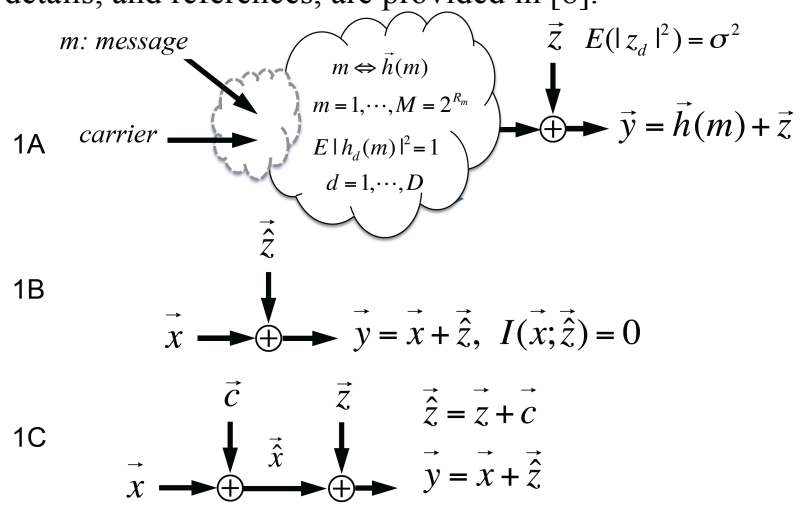

Fig.1: System Block Diagram

Acknowledgement: Author is thankful to Dr. H. Ebrahimzad for the simulations reported in this article.

\section{References:}

1. O. N. Alrabadi, A. Kalis, C. B. Papadias, R. Prasad," Aerial modulation for high order PSK transmission schemes," 1st International Conference on Wireless Communication, Vehicular Technology, Information Theory and Aerospace \& Electronic Systems Technology, VITAE 2009.

2. O. N. Alrabadi, A. Kalis, C. B. Papadias, R. Prasad,"A universal encoding scheme for MIMO transmission using a single active element for PSK modulation schemes",IEEE Transactions on Wireless Communications, Volume: 8 , Issue: 10, Page(s):5133 $-5142$

3. R. Bains, 'On the Usage of Parasitic Antenna Elements in Wireless Communication Systems', PhD Thesis

4. G. J. Foschini, "Layered space-time architecture for wireless communication in a fading environment when using multiple antennas," Bell Labs Syst. Tech. J., vol. 1, p. 41-59, 1996.

5. E. Telatar "Capacity of Multi-antenna Gaussian Channels", European Trans. on Telecomm10 (6): 585-595.

6. V. Tarokh, N. Seshadri, and A. R. Calderbank, "Space-time codes for high data rate wireless communication: Performance analysis and code construction". IEEE Trans. on Information Theory 44 (2): 744-765

7. L. Zheng and D. Tse, "Diversity and Multiplexing: A Fundamental Tradeoff in Multiple Antenna Channels", IEEE Transactions on Information Theory, vol. 49, May 2003, pp. 1073-96

8. A. K. Khandani, Media-based Modulation, Technical Report, Univ. Waterloo (cst.uwaterloo.ca / reports)

9. Edelman, Rao. Random matrix theory. Acta \# 14:233-297, 2005. 


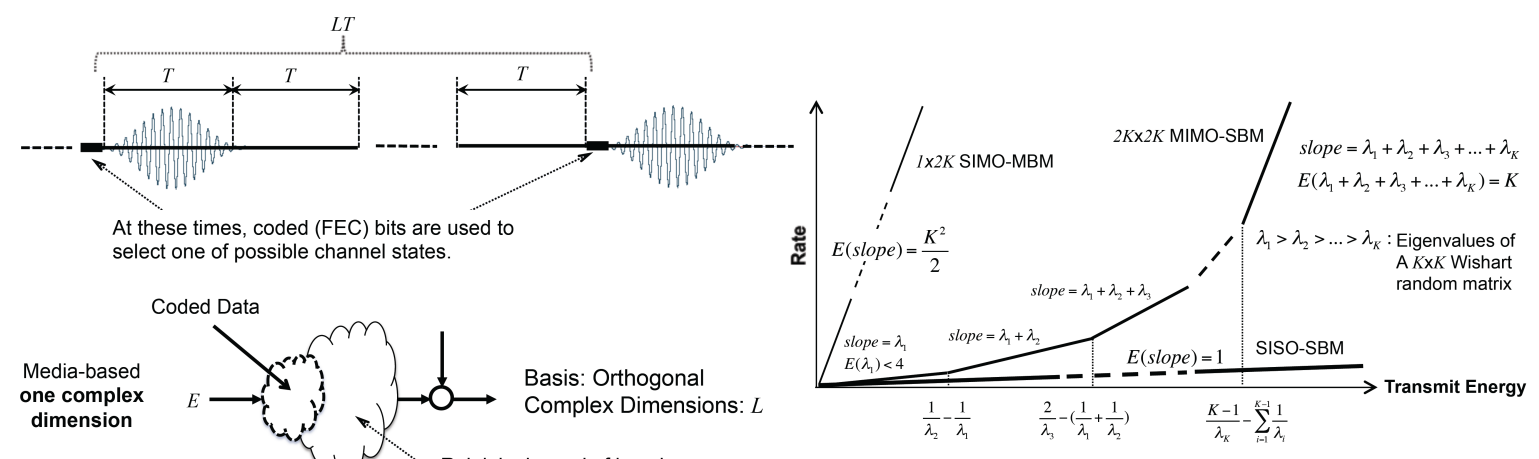

Raleigh channel of impulse

Fig. 2: Pulse Shaping in Time.

Fig.3: Effective Dimensionality of MBM vs. SBM.
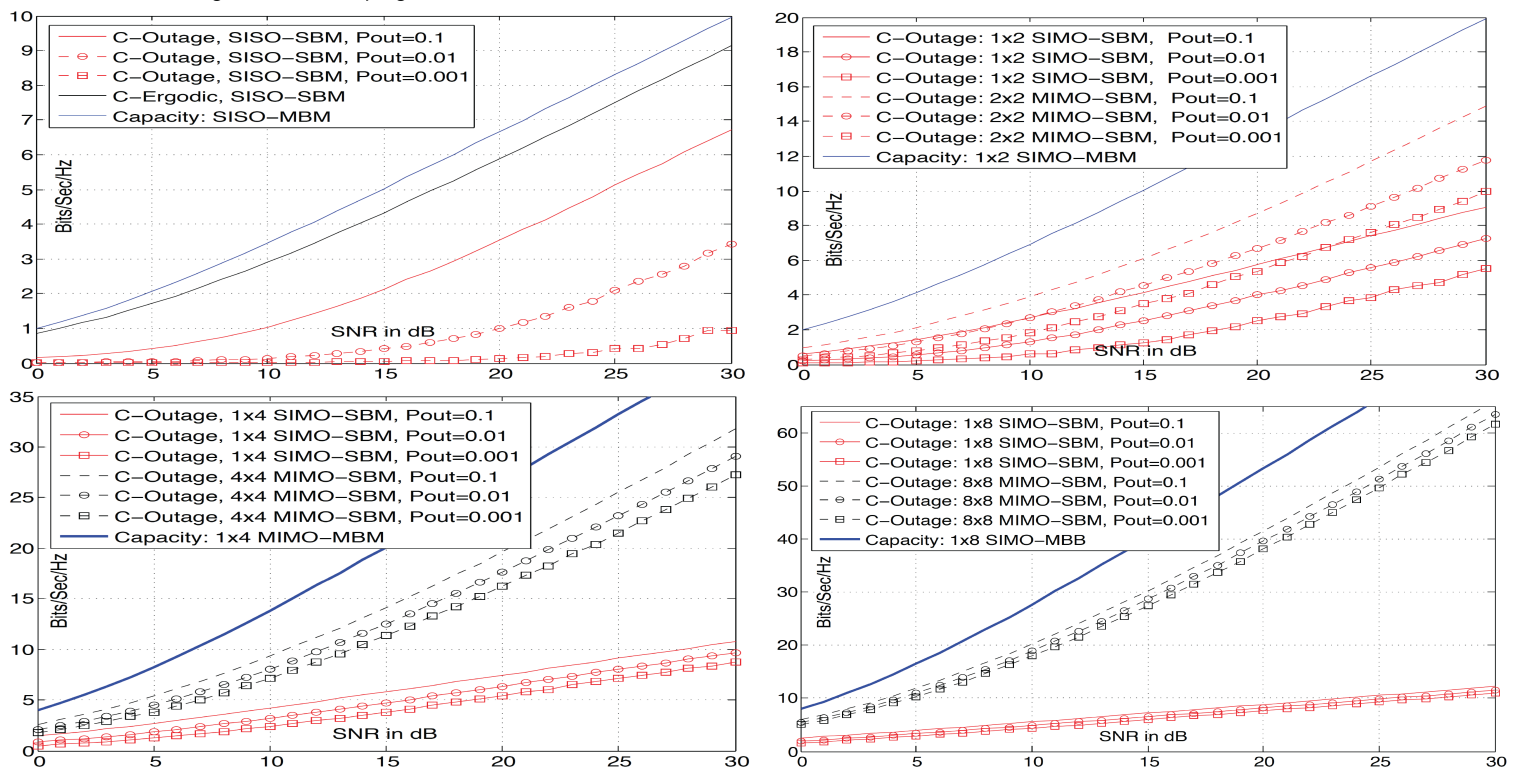

Fig.4: Comparisons vs. MIMO Outage Capacity.
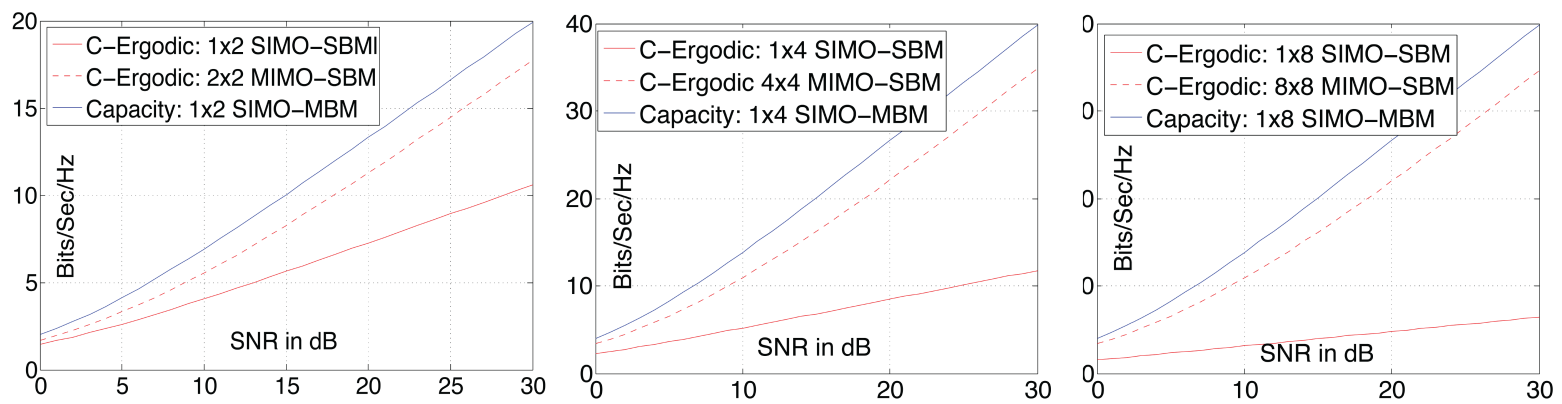

Fig.5: Comparisons vs. MIMO Ergodic Capacity (no water filling)

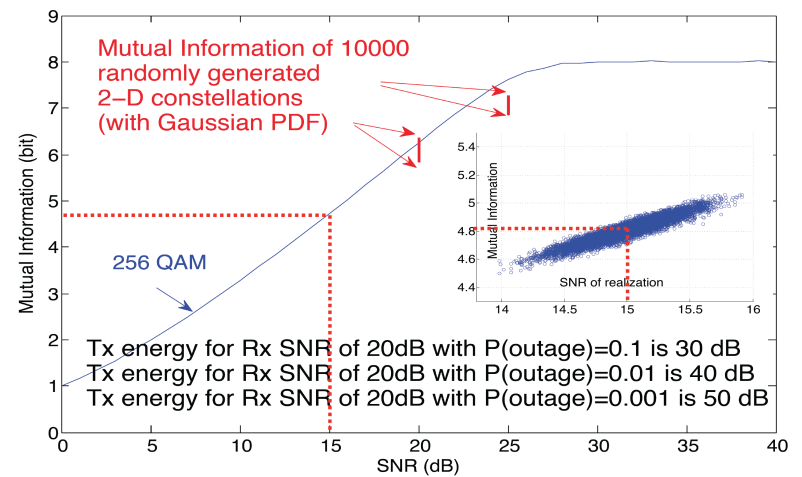

Fig. 6: SISO-MBM Random Constellations vs. 256 QAM.

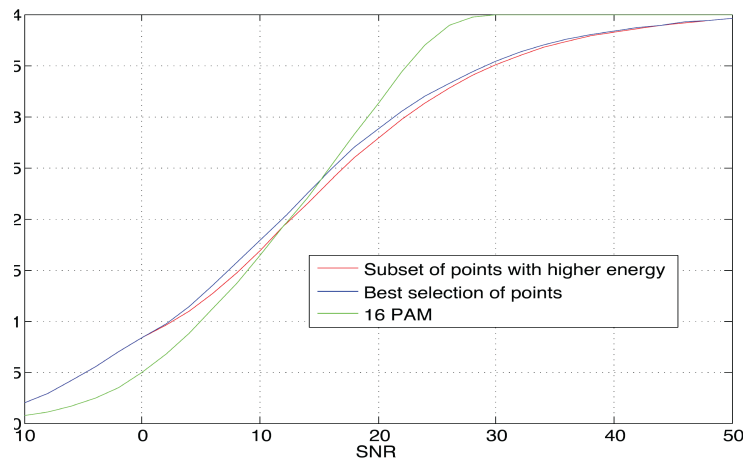

Fig. 7: Selection Gain 